\title{
Transjugular Intrahepatic Portosystemic Shunt for Portal Vein Cavernous Transformation: A Systematic Review and Single-Arm Meta-Analysis
}

\author{
Jingxin Yan ${ }^{a, b}$ Manjun Deng ${ }^{c}$ Yaxuan Wang ${ }^{d}$ Meilin Zhu ${ }^{e, f}$ Ting Li ${ }^{g, h}$ \\ Haiyang $\mathrm{Hu}^{\mathrm{b}, \mathrm{i}}$ Zhenwu Lei ${ }^{\mathrm{a}}$ Yingxing Guo ${ }^{\mathrm{a}}$ Lushun Zhang ${ }^{\mathrm{j}, \mathrm{k}}$
}

\begin{abstract}
aDepartment of Interventional Therapy, Affiliated Hospital of Qinghai University, Xining, China; ${ }^{b}$ Department of Postgraduate, Qinghai University, Xining, China; 'Department of Hepatopancreatobiliary Surgery, Affiliated Hospital

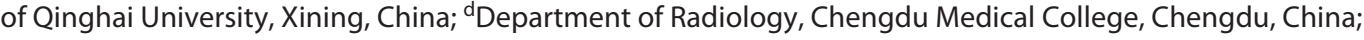
eSchool of Medicine, University of Electronic Science and Technology of China, Chengdu, China; fDepartment of Radiology, Sichuan People's Hospital, Chengdu, China; 'Department of Orthopedics, Sichuan People's Hospital, Chengdu, China; ' Department of Postgraduate, Chengdu Medical College, Chengdu, China; iDepartment of Oncology, Affiliated Hospital of Qinghai University, Xining, China; 'Department of Pathology and Pathophysiology, Chengdu Medical College, Chengdu, China; ${ }^{k}$ Center of Sichuan for Elderly Care and Health, Chengdu Medical College, Chengdu, China
\end{abstract}

\section{Keywords}

Transjugular intrahepatic portosystemic shunt · Portal vein cavernous transformation · Systematic review · Single-arm meta-analysis

\begin{abstract}
Background:Transjugular intrahepatic portosystemic shunt (TIPS) is technically challenging in the treatment of portal vein cavernous transformation (PVCT), and there is no highquality evidence regarding whether it is an option for patients with PVCT. We carried out a systematic review and meta-analysis to assess the feasibility and safety of TIPS for PVCT. Methods: Systematic search of PubMed, Chinese National Knowledge Infrastructure (CNKI) database, Cochrane Library, Embase, and Wanfang database through December 2021 for appropriate studies reporting efficacy and safety in patients with PVCT undergoing TIPS. The main outcome included the technical success rate, postoperative rebleeding rate, postoperative hepatic encephalopathy rate, stent pa-
\end{abstract}

tency rate, preoperative, and postoperative portal pressure. Results: Ten studies, including 292 patients were included. Our results showed that TIPS was technically successful in 82.97\% (95\% confidence interval [CI]: 77.14\%-88.41\%, $p=$ $0.297)$ with low heterogeneity $\left(I^{2}=18.39 \%, p=0.279\right)$. Postoperative rebleeding occurred in $9.56 \%(95 \% \mathrm{Cl}: 4.55 \%-$ $16.77 \%, p=0.073)$ with moderate heterogeneity $\left(I^{2}=46.45 \%\right.$, $p=0.06)$. Postoperative hepatic encephalopathy occurred in 18.55\% (95\% Cl: $9.23 \%-27.05 \%, p=0.343$ ) with moderate heterogeneity $\left(I^{2}=48.62 \%, p=0.049\right)$. Stent patency during follow-up was in $78.43 \%$ (95\% Cl: $70.74 \%-85.20 \%, p=0.805)$ with low heterogeneity $\left(I^{2}=0 \%, p=0.654\right)$. Postoperative portal pressure significantly reduced $(\mathrm{WMD}=12.79 \mathrm{~mm} \mathrm{Hg}$, $95 \% \mathrm{Cl}: 12.09-13.48 \mathrm{~mm} \mathrm{Hg}, p<0.00001)$ with high heterogeneity $\left(I^{2}=61.4 \%, p=0.02\right)$. Both Begg test and funnel plot showed that there was no significant publication bias. Conclusions: TIPS is feasible and safe in patients with PVCT and PVCT should not be considered an absolute contraindication to TIPS.
Karger@karger.com www.karger.com/ddi Karger"
(C) 2022 S. Karger AG, Base
Correspondence to:

Jingxin Yan, jingxinyan1997@ 126.com Lushun Zhang, zhangls2012@163.com 


\section{Introduction}

Portal vein cavernous transformation (PVCT) is characterized by long-term portal vein thrombosis (PVT), interconnected periportal and intrahepatic collateral veins, leading to portal hypertension $[1,2]$. In addition, patients with PVCT usually have a history of cirrhosis or other liver diseases. PVCT increases the incidence of bleeding, refractory ascites, and hypersplenism. If left untreated, PVT and PVCT may lead to life-threatening mesenteric venous gangrene and other serious complications $[3,4]$. However, there is no consensus regarding the application of interventional therapy in patients with PVCT, so the therapeutic option is rather limited and effective treatment is needed to be explored.

Transjugular intrahepatic portosystemic shunts (TIPSs) are an effective method in the management of portal hypertension, variceal bleeding, and refractory ascites $[5,6]$, with lower morbidity and mortality compared to surgical shunting. Recently, the Baveno VI consensus recommended that TIPS is an effective treatment of portal hypertension caused by various causes and TIPS can significantly decrease postoperative rebleeding rate and mortality [7]. TIPS is also considered as a safe and effective method in the treatment of PVT, when technically feasible, as it prevents the extension of thrombosis and lows the risk of patients with PVCT. However, an evidence-based review concluded that PVCT is the main determinant of surgical failure [8], so TIPS for PVCT is still a challenge.

Previous studies reported that TIPS was a feasible, effective, and challenging treatment for patients with PVCT $[9,10]$. These studies prompted us to investigate a safe and effective therapy that could improve the prognosis of patients with PVCT. Although some conclusions have been made, the safety and effect of TIPS have not been systematically reviewed. Therefore, we performed a meta-analysis to assess the existing evidence for the technical feasibility, safety, and efficacy of TIPS for patients with PVCT.

\section{Method}

\section{Literature Search}

This study was carried out and reported in accordance with the Preferred Reporting Items for Systematic Reviews and Meta-Analyses (PRISMA) guidelines [11] (PRISMA checklist available in online suppl. material; for all online suppl. material, see www. karger.com/doi/10.1159/000522313). Comprehensive research of databases was performed to collect the trails about TIPS in the treatment of PVCT. Only randomized controlled trials, cases reports, cases series, retrospective studies, and single-arm studies were included. Language restriction was English and Chinese. We searched articles published up to December 2021, including PubMed, CNKI, Cochrane Library, Embase, and Wanfang database. The search strategy included following database subject headings and abstract words: "Transjugular intrahepatic portosystemic shunt" OR "TIPS" OR "interventional treatment," "portal vein cavernous transformation" OR "cavernous transformation of the portal vein." And reference lists from the resulting publications and reviews were searched.

\section{Study Selection}

All citations were assessed by two authors independently, and the other two authors checked the articles retrieved according to inclusion and exclusion criteria. Disagreement was settled by discussion and consensus. Study titles and abstracts were inspected firstly, and the full texts of potentially eligible studies were reviewed further by the same two authors. Studies were considered eligible if they met the criteria as follows: (1) design: randomized controlled trial, cases reports, cases series, retrospective studies, and single-arm studies; (2) participants: patients were diagnosed with PVCT; (3) patients underwent TIPS. The number of studies and reasons for exclusion were as follows: (1) valid data were unavailable or data not completed or inaccurate so that the study could not be analyzed; (2) relevant outcome indexes were not reported; (3) comments, reviews, abstracts, editorials, and thesis; and (4) duplicate publications.

\section{Data Extraction}

According to the inclusion and exclusion criteria, we read the full text, excluded the literature that does not accord with this research scheme, and record the reasons and quantity of exclusion. Finally, the selected literature was cross-checked by two researchers. The following items of information were extracted: the name of the first author, year of publication, baseline characteristics of patients, sample size, and other prognostic information or relevant information.

\section{Risk Assessment}

The Newcastle-Ottawa scale (NOS) scores were adopted to evaluate observational studies [12]. Studies with a mean score $\geq 5$ were included. Quality assessment was conducted by two independent reviewers and in case of conflict, conflict was resolved by consensus. The methodological quality of case series and case reports was measured by the "Methodological quality and synthesis of case series and case reports" [13].

\section{Statistical Analysis}

We performed a Bayesian meta-analysis to measure the incidence rate of technical successful rate, postoperative rebleeding rate, postoperative hepatic encephalopathy rate, and stent patency rate. The hierarchical model was used for Bayesian meta-analysis and estimating pooled prevalence. Complete Bayesian analysis was conducted using Markov Chain Monte Carlo simulation of posterior distribution, and the results were based on 200,000 iterations after a burn-in period of 50,000 iterations. All the analyses were generated by Rstudio and R (4.0.2) using "xlsx" and "R2jags" and "mcmcplots" and "dplyr" and "forestplot" packages [14, 15]. In addition, the $95 \%$ highest posterior density and $95 \%$ credible inter- 


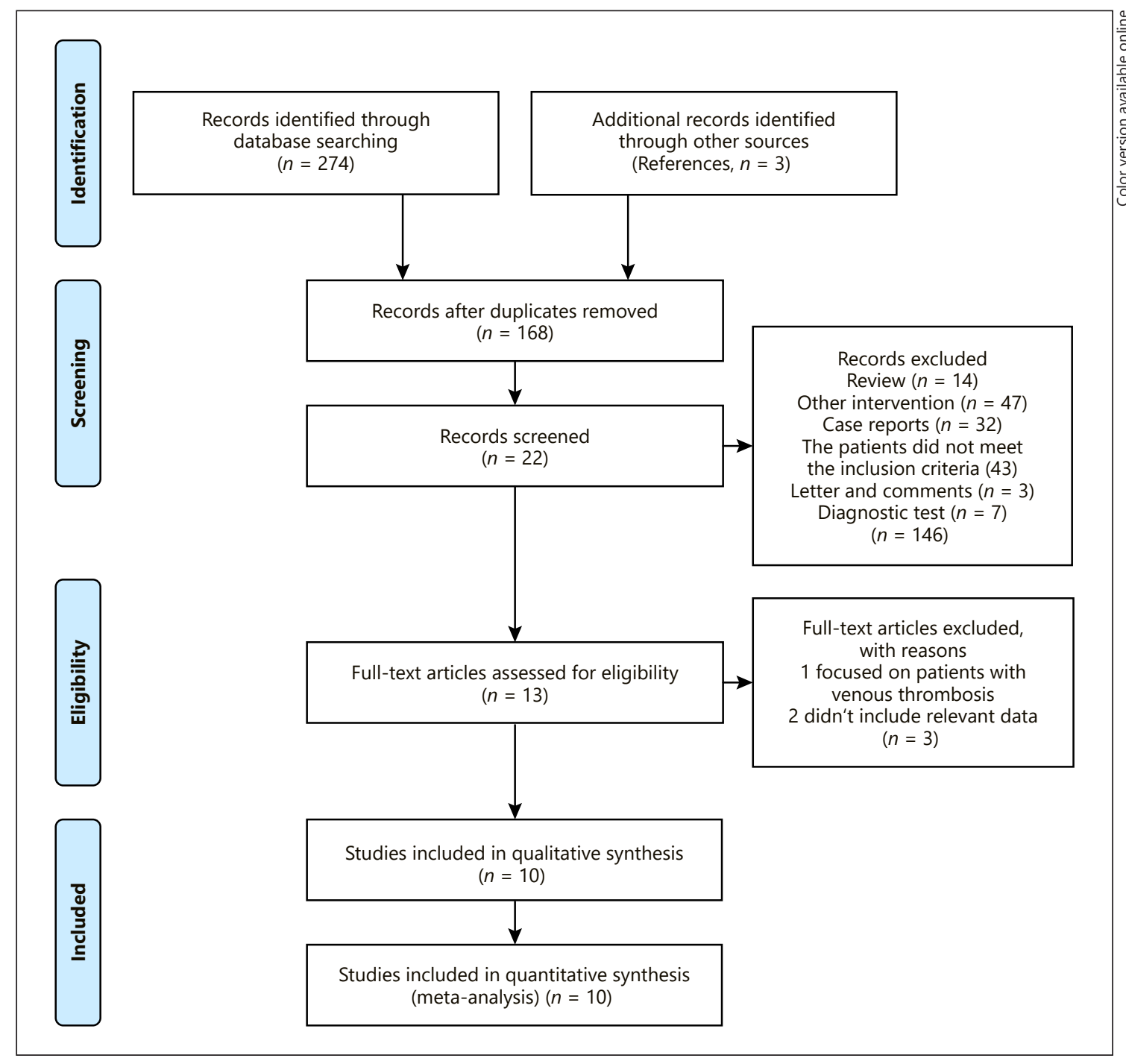

Fig. 1. Flow diagram.

vals (CI) were computed. Then, as recommended in Bayesian inference, sensitivity analysis for the choice of the prior was performed choosing between plausible possibility [16] and excluding skeptical and enthusiastic priors. The double arccosine transformation was used to build the funnel plot [17]. Online supplementary material 1 shows model implementation in $\mathrm{R}$ language.

Changes between preoperative and postoperative portal pressure were assessed using mean difference and 95\% CI. For these continuous data, we performed a random-effect model meta-analysis when significant heterogeneity was present.

To objectively evaluate the publication bias of the included literatures, the Begg test with $p<0.05$ as the existence of publication bias was performed, the values larger than which were considered as no publication bias. Statistical heterogeneity between the studies in effect measures will be assessed by Mantel-Haenszel $\chi^{2}$ test and the $I^{2}$ statistic. We considered possible explanations where substantial heterogeneity $\left(I^{2}\right.$ statistic above $\left.50 \%\right)$ was detected and where applicable. In addition, if there was substantial heterogeneity $\left(I^{2} \geq 50 \%\right)$, the trials were heterogeneous; meta-regression, subgroup analysis, and sensitivity analysis were selected to explore the source of the heterogeneity. Stata 16.0 was used to conduct a metaregression to investigate potential sources of variability in prevalence reported between studies [18].

\section{Results}

\section{Studies Selection and Characteristics}

The initial search yielded 274 records. After a comprehensive screening, 13 potentially eligible studies were identified. According to the inclusion criteria, 10 studies [19-28] (4 studies published in Chinese [20, 23, 27, 28] 


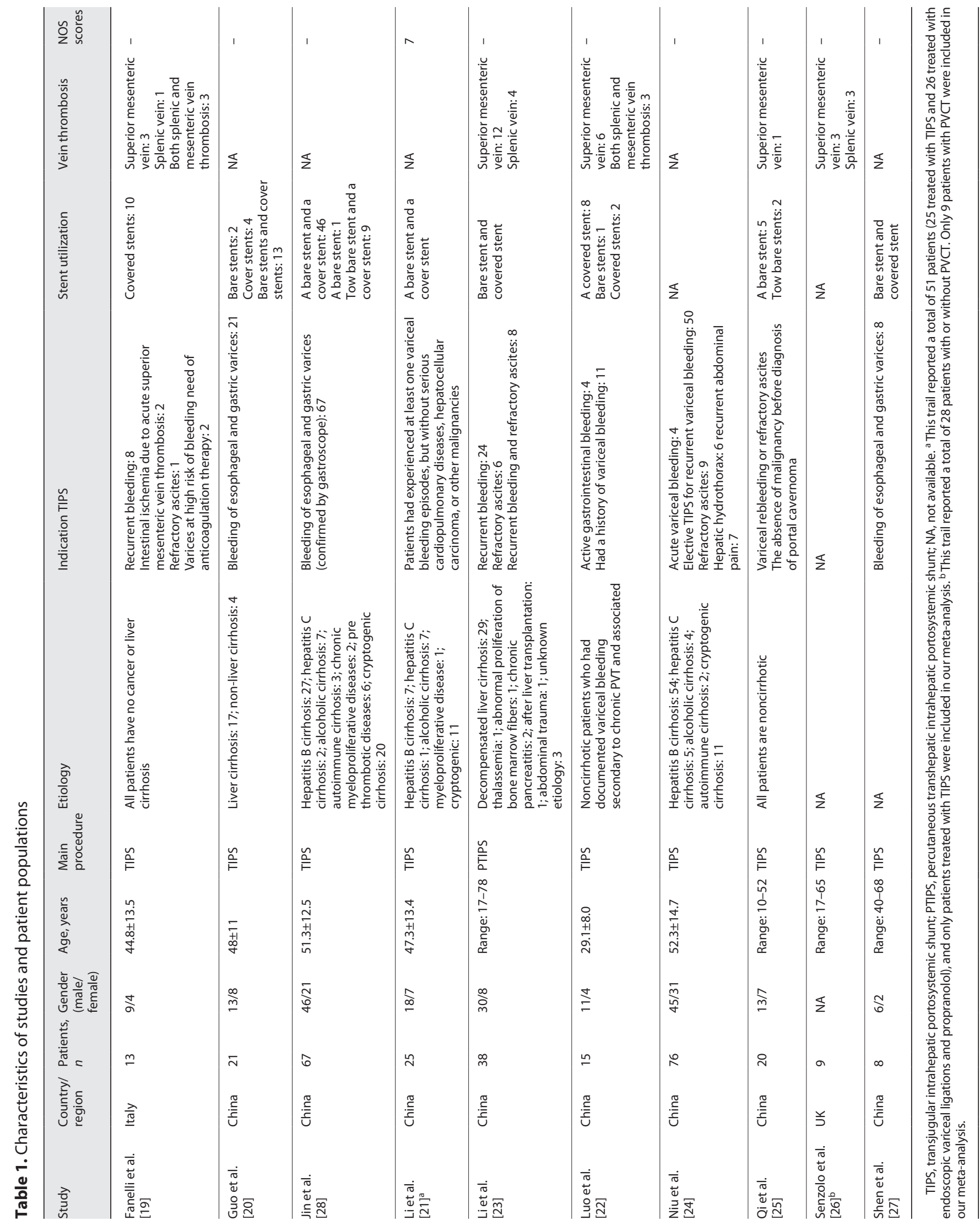




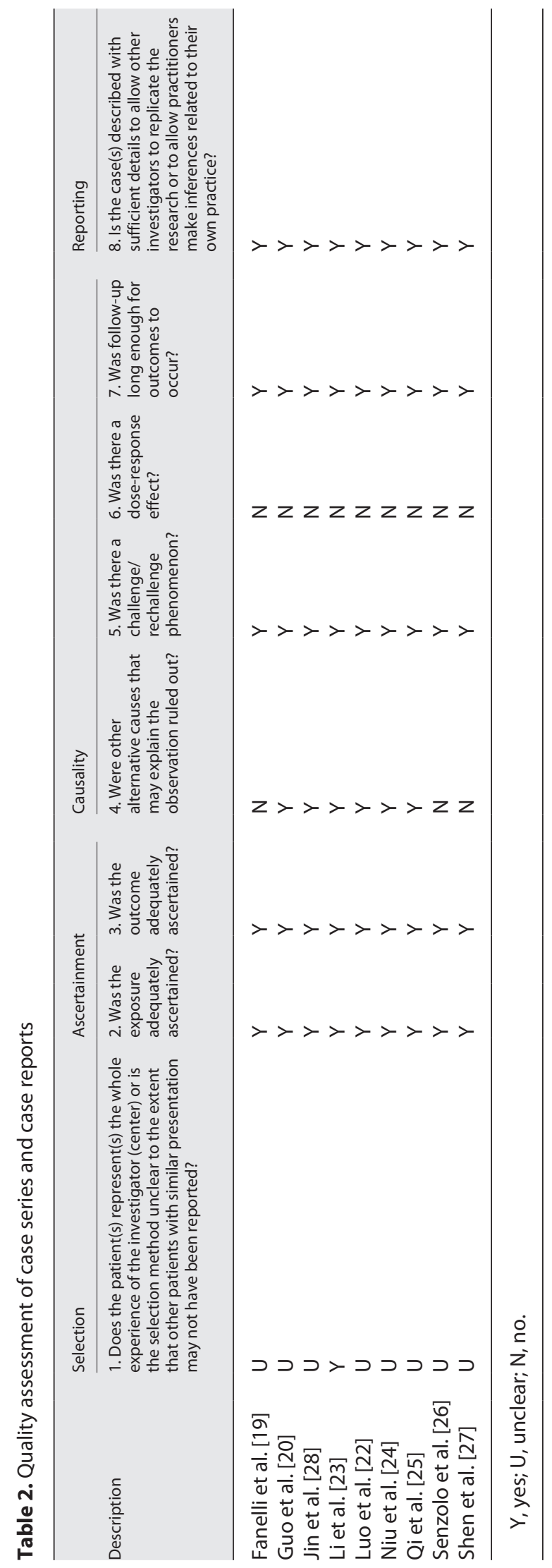

and 6 studies published in English [19, 21, 22, 24-26]) were included in our meta-analysis. Figure 1 displays the selection algorithm and numbers of included and excluded studies.

Overall, our meta-analysis included 10 studies that reported TIPS or percutaneous transhepatic intrahepatic portosystemic shunt (PTIPS) in the treatment of PVCT. The sample size ranged from 8 to 76 , reaching a total of 292 cases. Nine studies provided data on the indication of TIPS, 8 studies provided data on the etiology, and 6 provided data regarding the vein thrombosis. The main study characteristics of included studies are shown in Table 1 and the quality assessment of case series and case reports are shown in Table 2.

\section{Meta-Analysis Results}

Successful Rate

All included studies reported the technically successful rate. The pooled successful rate was $79.64 \%$ (95\% CI: $67.28 \%-89.33 \%)$ and significant heterogeneity can be identified $\left(I^{2}=68.67 \%, p=0.06\right)$. Moreover, we performed a sensitivity analysis to explore the potential source of heterogeneity. When Qi's [25] study was excluded, we found that there is a low heterogeneity $\left(I^{2}=\right.$ $18.39 \%, p=0.279$ ) between included studies and the pooled successful rate was $82.97 \%$ (95\% CI: $77.14 \%-$ 88.41\%; Fig. 2). Both Begg test ( $p=0.325)$ and funnel plot (Fig. 3) showed that there was no significant publication bias.

\section{Postoperative Rebleeding Rate}

Nine included studies [19-25, 27, 28] reported the postoperative rebleeding rate. The pooled postoperative rebleeding rate was 9.56\% (95\% CI: $4.55 \%-16.77 \%$; Fig. 4) with moderate heterogeneity $\left(I^{2}=46.45 \%, p=0.06\right)$.

Incidence of Postoperative Hepatic Encephalopathy

Nine included studies [19-25, 27, 28] reported the postoperative hepatic encephalopathy rate. The pooled postoperative hepatic encephalopathy rate was $18.55 \%$ (95\% CI: 9.32\%-27.05\%; Fig. 5) with no significant heterogeneity $\left(I^{2}=48.62 \%, p=0.049\right)$.

Stent Patency Rate during Follow-Up

Eight included studies [19-23, 25, 27, 28] reported the stent patency rate. The pooled stent patency rate was 78.43\% (95\% CI: 70.43\%-85.20\%; Fig. 6) with moderate heterogeneity $\left(I^{2}=0 \%, p=0.654\right)$. 


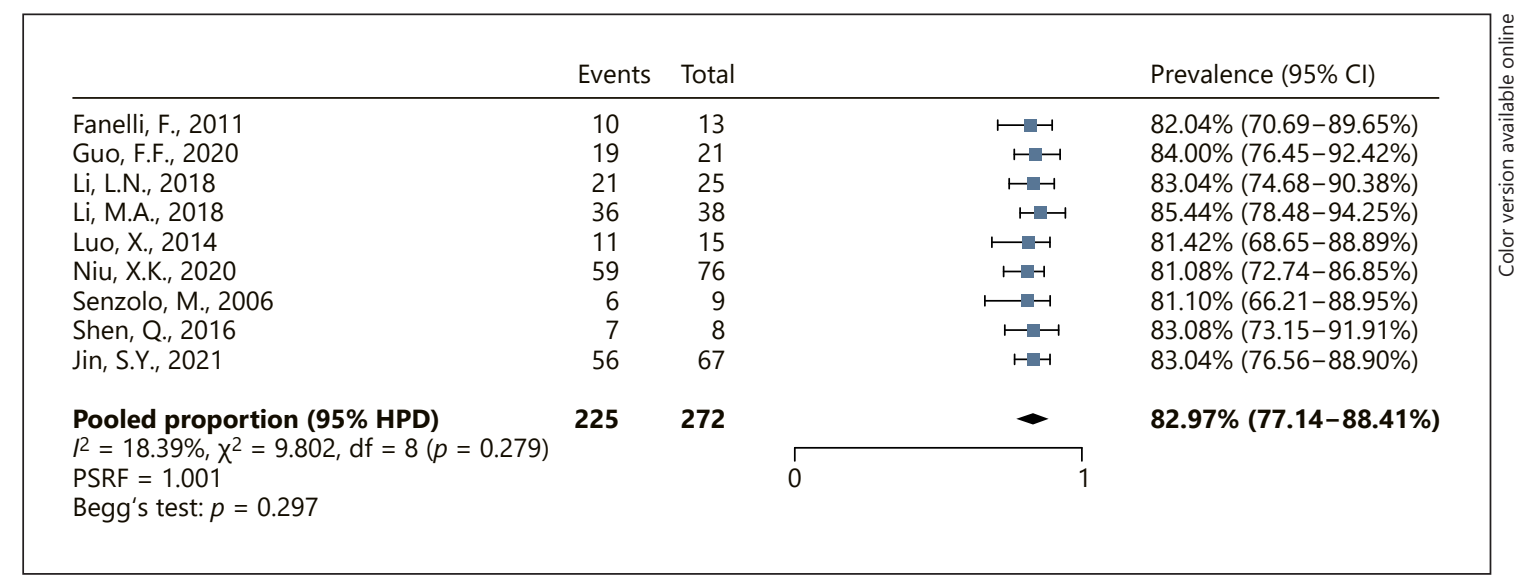

Fig. 2. A forest plot showing the success rate of TIPS on PVCT.

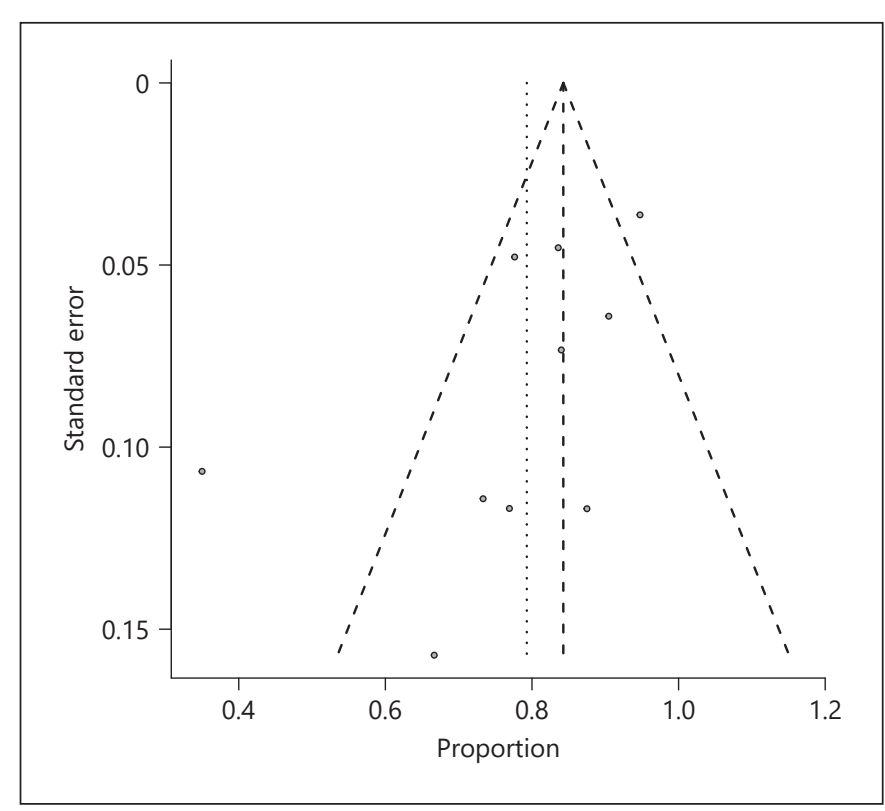

Fig. 3. A funnel plot showing the publication bias.

Changes of Preoperative and Postoperative Portal Pressure

Six included studies $[19,20,22,23,25,28]$ reported the differences between preoperative and postoperative portal pressure. Preoperative portal pressure ranged from 22.9 to $28.09 \mathrm{~mm} \mathrm{Hg}$ with a mean preoperative portal pressure of $25.30 \mathrm{~mm} \mathrm{Hg}$. The postoperative portal pressure significantly reduced (WMD = $12.79 \mathrm{~mm} \mathrm{Hg}$, 95\% CI: 12.09$13.48 \mathrm{~mm} \mathrm{Hg}$; Fig. 7) with significant heterogeneity $\left(I^{2}=\right.$ $61.4 \%)$, so a random-effect model was performed.

TIPS for Portal Vein Cavernous Transformation
Survival Rate

Seven included studies $[21-25,27,28]$ reported the survival rate in follow-up. All those studies reached a total survival rate of $80 \%$. Jin et al. [28] reported the 1 - and 2 -year survival rates are $94.3 \%$ and $83.2 \%$, respectively, and Niu et al. [24] reported the 1- and 2-year survival rates are $93.7 \%$ and $83 \%$, respectively, indicating patients with PVCT underwent TIPS can reach a favorite clinical effect. The main outcomes are shown in Table 3.

\section{Meta-Regression and Subgroup Analysis}

Nine studies reported the male/female ratio; all studies reported the main procedure, publication year, and countries/region; and six studies reported that all the patients with or without cirrhosis, so all those factors were taken into account to perform a meta-regression. Unfortunately, we found that successful rate (online suppl. Table S1), postoperative hepatic encephalopathy rate (online suppl. Table S2), postoperative rebleeding rate (online suppl. Table S3), and stent patency rate (online suppl. Table S4) were not significantly associated with male/female ratio, main procedure, countries/region, publication year, and cirrhosis. We found that changes between preoperative and postoperative portal pressure is weakly associated with publication year $[\exp (b)=0.72,95 \%$ CI: 0.54-0.95] and cirrhosis $[\exp (\mathrm{b})=0.96,95 \% \mathrm{CI}$ : 0.009-0.99] (online suppl. Table S5). However, the weak association should be identified by further studies.

Cirrhosis, technical differences, study design, and country/region were taken into account to perform a subgroup analysis (online suppl. Table S6). We found that it is not yet appropriate to draw final conclusions about the impact of technical differences, study design, and coun- 


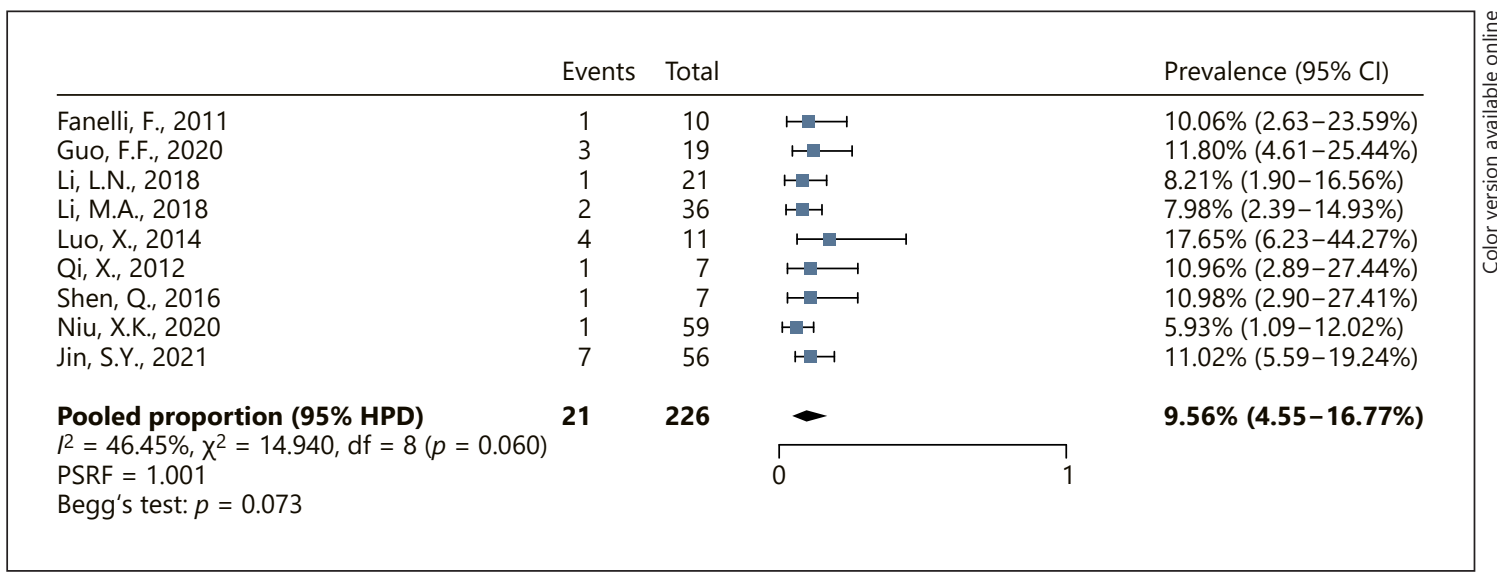

Fig. 4. A forest plot showing the postoperative rebleeding rate of TIPS on PVCT.

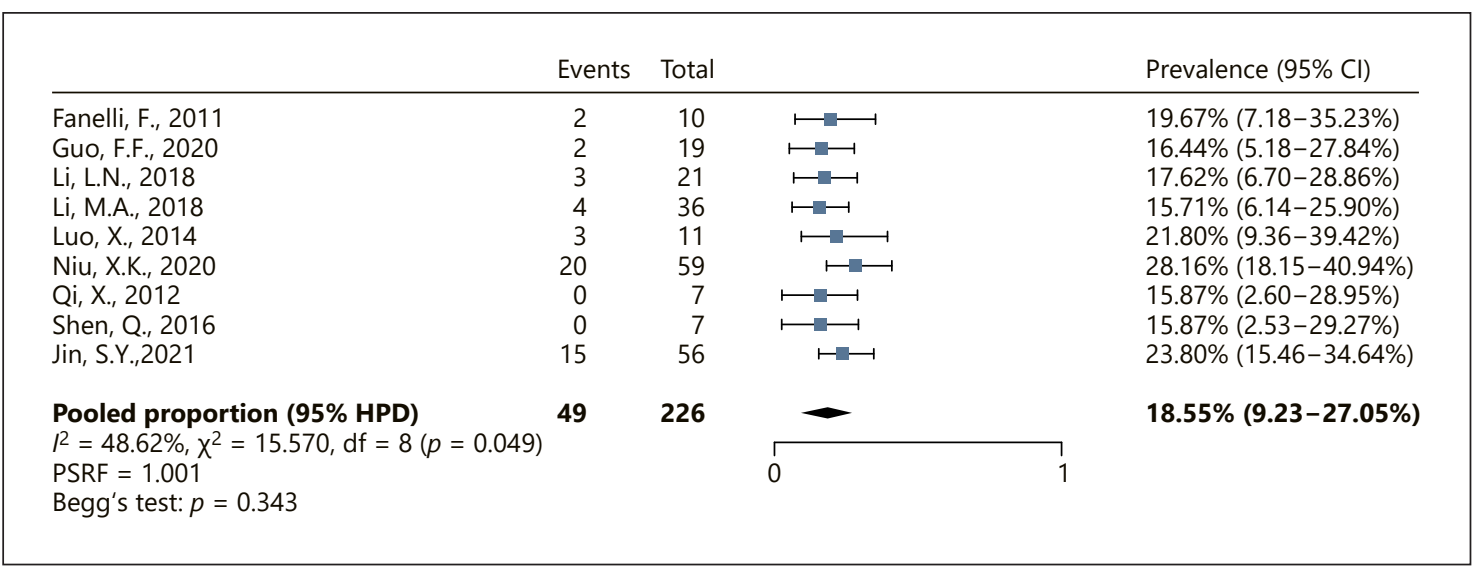

Fig. 5. A forest plot showing the postoperative hepatic encephalopathy rate of TIPS on PVCT.

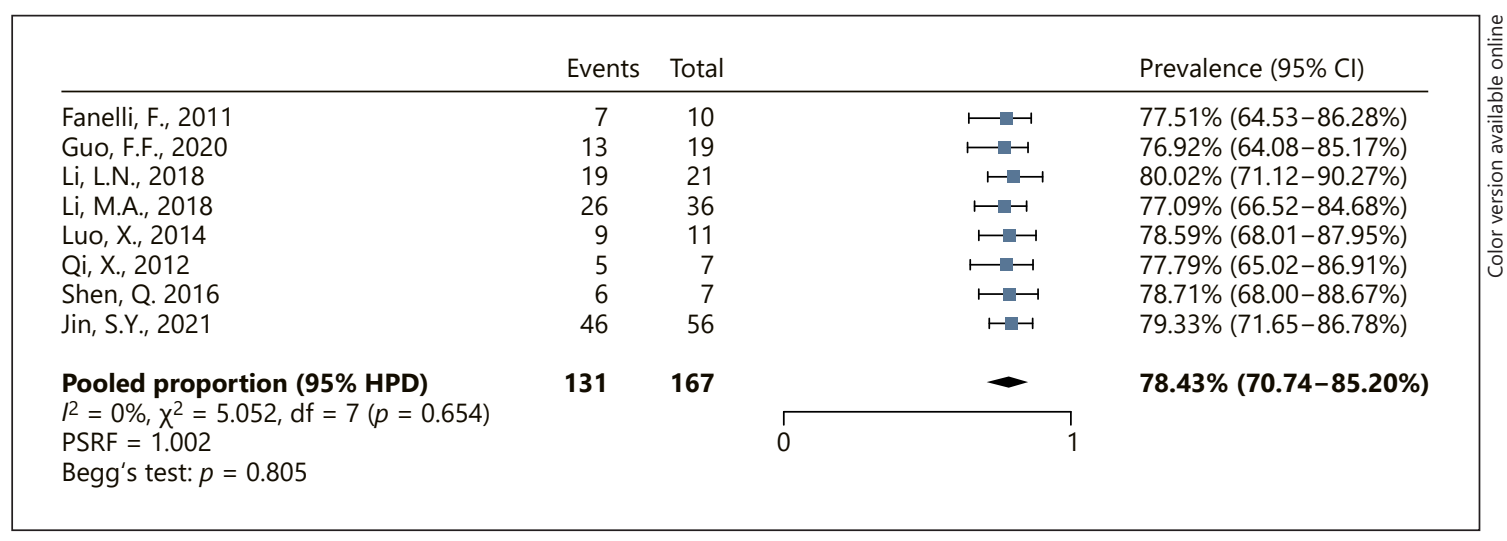

Fig. 6. A forest plot showing the stent patency rate of TIPS on PVCT. 


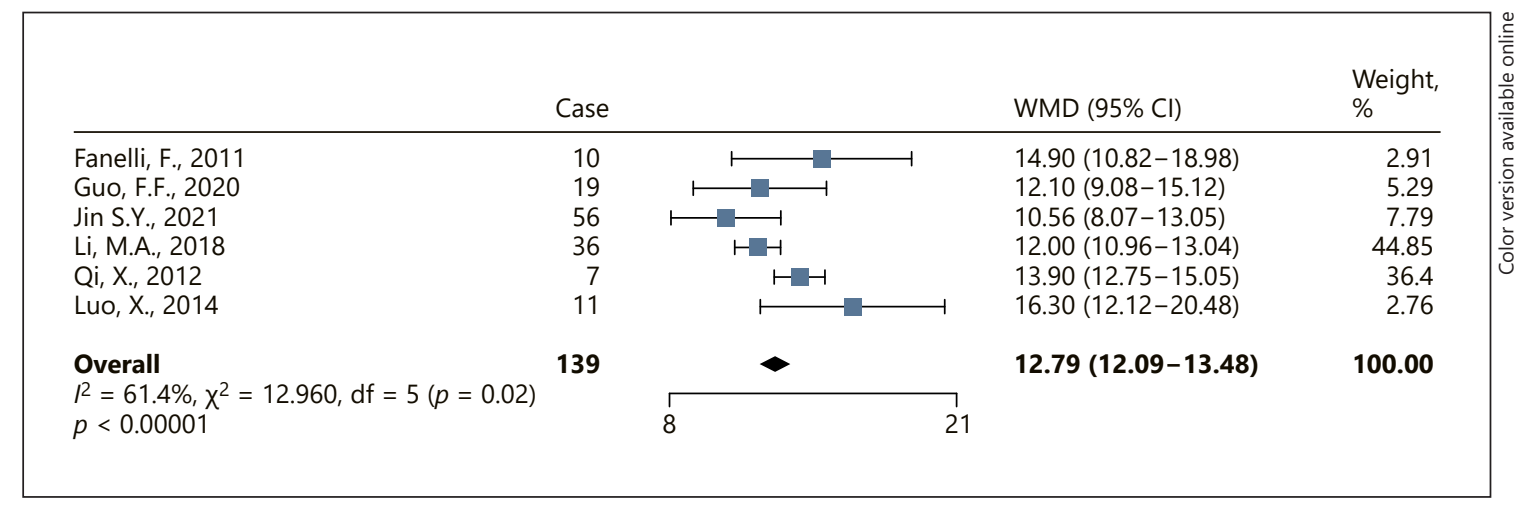

Fig. 7. A forest plot showing the changes of preoperative and postoperative portal pressure of TIPS on PVCT.

Table 3. Characteristics and main outcomes

\begin{tabular}{|c|c|c|c|c|c|c|c|c|}
\hline Fanelli et al. [19] & Mean $17.4 \pm 14.7$ (range: $3.3-40.1$ ) & NA & $10 / 13$ & $1 / 10$ & $2 / 10$ & $7 / 10$ & $22.9 \pm 6$ & $8 \pm 2.7$ \\
\hline Guo et al. [20] & Range: 3-24 & NA & $19 / 21$ & $3 / 19$ & $2 / 19$ & $13 / 19$ & $23.5 \pm 4.6$ & $11.4 \pm 4.9$ \\
\hline Li et al. [21] & Mean: 21 (range: 1-47) & $85.7 \%$ & $21 / 25$ & $1 / 21$ & $3 / 21$ & $19 / 21$ & NA & NA \\
\hline Li et al. [23] & Mean: $26.7 \pm 10.4$ (range: $3.0-74.0$ ) & $80.6 \%$ & $36 / 38$ & $2 / 36$ & $4 / 36$ & $26 / 36$ & $25.2 \pm 2.9$ & $13.2 \pm 1.3$ \\
\hline Luo et al. [22] & Median follow-up time was 45.2 & 80 & $11 / 15$ & $4 / 11$ & $3 / 11$ & $9 / 11$ & $25.8 \pm 5.7$ & $9.5 \pm 4.2$ \\
\hline Niu et al. [24] & Mean: $45.9 \pm 23.6$ & $\begin{array}{l}1 \text { year: } 93.7 \% \\
2 \text { years: } 83.0 \%\end{array}$ & $59 / 76$ & $1 / 59$ & $20 / 59$ & NA & NA & NA \\
\hline Qi et al. [25] & $\begin{array}{l}\text { Median follow-up time was } 19.9 \text { (range, } \\
\text { 3.9-96) }\end{array}$ & $90 \%$ & $7 / 20$ & $1 / 7$ & $0 / 7$ & $5 / 7$ & $26.3 \pm 1.1$ & $12.4 \pm 1.1$ \\
\hline
\end{tabular}

NA, not available.

try/region on successful rate, postoperative hepatic encephalopathy rate, postoperative rebleeding rate, stent patency rate, and changes between preoperative and postoperative portal pressure, because only one study used PTIPS and only two studies were from non-China areas. Besides, a subgroup analysis of with or without cirrhosis may reduce $I^{2}$ statistics value on changes between preoperative and postoperative portal pressure, stent patency rate during follow-up, and postoperative rebleeding rate. However, heterogeneity of incidence of postoperative hepatic encephalopathy and successful rate cannot be explained completely by cirrhosis, so further studies are needed to explore the heterogeneity.

TIPS for Portal Vein Cavernous Transformation

\section{Discussion}

In our study, ten studies, including 292 patients with PVCT were included. Pooled results demonstrated that TIPS is feasible in most patients with PVCT and TIPS could be a choice for those who with PVCT uncontrolled by conventional therapy such as nonselective $\beta$-blocker, endoscopic variceal ligation [29], and vasoactive drugs (somatostatin or its analog, octreotide; vasopressin or its analog, terlipressin) [30].

A recent study by Lomax and colleagues [9] concluded that PVCT should not be considered an absolute contraindication to TIPS, although the presence of PTCV is as- 
sociated with a significantly high failure rate (high-quality evidence) and PVCT was previously considered as a contraindication to TIPS because of its postoperative adverse events and technical difficulty $[31,32]$. Besides, the experiences from TIPS in the treatment of PVCT are exceedingly limited due to low incidence of PVCT, and only a few cases were reported. Specifically, it is tough to create an intrahepatic shunt at the same time as avoiding damage to surrounding cavernous vein [33]. In other words, the most important and challenging step of TIPS is the shunt creation, which establishes a channel between the hepatic vein and the portal vein, but PVCT complicates the vascular condition, leading to the difficulties of TIPS. Even previous studies concluded that TIPS is exceedingly feasible for PVT recanalization [34], PVCT is still the main determinant of the technical success because portal vein and its branches are gradually thrombosed or occluded in this setting. In patients with portal vein occluded completely, when TIPS was performed, the blood could not be drawn back, so it was difficult to judge whether the shunt creation was successful. In this setting, ultrasound-guided percutaneous transhepatic or percutaneous transsplenic approach is also helpful to find an appropriate puncture target $[35,36]$.

According to previous studies, percutaneous transhepatic or transsplenic approach could be feasible in finding an appropriate site for TIPS creation [37-40], making surgery much easier during treatment of PVCT. In our meta-analysis, only one included a study by Li et al. [23] reported that PTIPS was used, achieving a low postoperative rebleeding rate $(2 / 36)$ and acceptable successful rate $(36 / 38)$. However, further trials are needed to verify the safety and efficacy of PTIPS and other possible approaches.

To our knowledge, the feasibility of TIPS placement has been reported to be beneficial in patients with cirrhosis and PVT [26, 41-43]. Compared to surgery, TIPS is a technique with relatively lower invasiveness, which can be performed in vulnerable patient groups, reducing the postoperative portal pressure. Besides, a recent metaanalysis by Rodrigues et al. [8] involving 392 patients, in which all patients had PVT (17\% with PVCT), concluded that TIPS was technically successful in 95\% and portal vein recanalization rate at 12 months is $79 \%$. Although Rodrigues et al. [8] had made some conclusions, there is no specific data regarding the TIPS for PVCT. Our results show that TIPS implantation can be successful in most cases $(82.97 \%$, 95\% CI: $77.14 \%-88.41 \%)$. Something should be noticed that one of our included studies by Qi et al. [25] reported a low success rate of $35 \%$ and it is sen- sitive to our pooled result. In Qi's [25] study, this difference can be explained by the fact that the main portal vein was occluded and became fibrotic cord in most patients $(19 / 20)$, indicating that occluded main portal vein makes TIPS creation more difficult. This finding was consistent with previous studies by Wils et al. [44] and Han et al. [45].

The stent patency rate is another important issue that should be noticed. When TIPS was performed, shunts patency can be achieved, and the expansion of thrombus can be avoided [46]. According to the study involving 392 PVT cases by Rodrigues et al. [8], shunt patency at 12 months was $84 \%$ (95\% CI: 76\%-90\%). Similarly, the stent patency rate during the follow-up in our meta-analysis was $78.43 \%$ (95\% CI: 70.43\%-85.20\%). After TIPS, anticoagulation may prevent thrombus extension and maintain PTV canalization $[47,48]$. However, among included studies, all patients received intraoperative anticoagulation, but data regarding postoperative anticoagulation were not reported. Interestingly, Wang et al. [49] thought that it is widely accepted that post-TIPS anticoagulation does not correlate with the stent patency, but Rodrigues et al. [8] thought that utilization of post-TIPS anticoagulation was associated with a higher patency rate. Besides, the patency of bare stent and covered stent is different and covered stent is generally considered as a technology with much more advantage for shunts patency [50-53]. There are still some controversies about the utilization of postTIPS anticoagulation in preventing thrombus extension and stent restenosis.

Considering the safety, postoperative hepatic encephalopathy rate and postoperative rebleeding rates are 18.55\% (95\% CI: 9.32\%-27.05\%) and 9.56\% (95\% CI: $4.55 \%-16.77 \%)$, respectively. Besides, according to the postoperative hepatic encephalopathy rate and postoperative rebleeding rate, we concluded that TIPS creation was generally safe, suggesting that TIPS is a feasible and effective procedure in the treatment of PVCT. In included studies, data regarding survival rate is limited (only 5 included studies reported survival rate), so it is not yet appropriate to draw final conclusions about survival rate. However, in an interventional catheter room, clinicians still need to be cautious, because the complex interconnected periportal or intrahepatic collateral veins add the difficulty of surgery.

Meta-regression and subgroup analysis were performed to find the resource of heterogeneity. We found that cirrhosis is a study-level factor that has weak association with our results. Francoz et al. [54] reported that PVCT may often be accompanied by cirrhosis. Besides, the prognosis and clinical outcome are different between 
patients with or without PVCT [55]. Shen et al. [56] found that liver stiffness significantly increased in PVCT patients with cirrhosis. Previous studies found that liver stiffness is closely related to the prognosis of patients with cirrhosis $[57,58]$, so we thought that the association between our results and cirrhosis can be explained by aforementioned studies. However, although the various factors can lead to PVCT, the etiology is not clear. Further studies are needed to verify this finding.

In this meta-analysis, Bayesian meta-analysis was performed. Compared to the frequentist meta-analysis, Bayesian meta-analysis provides more accurate estimates for small samples because this method takes into account possible bias, reaching more accurate estimates for small samples.

Similar to any meta-analyses, our study has some limitations. Firstly, single-arm studies and controlled studies were included in this meta-analysis, so the pooled results and final conclusions should be treated with cautions. Secondly, four included studies published in Chinese. For doctors outside of China, there will be some difficulties to understand these studies because of language. However, all Chinese studies are published in top Chinese journals and these results are also crucial for this meta-analysis. Thirdly, surgical experience and hospital cost should be taken into account in further studies. Fourthly, after meta-regression and subgroup analysis, we found that changes between preoperative and postoperative portal pressure are weakly associated with publication year and cirrhosis. We also found that cirrhosis may have an impact on changes between preoperative and postoperative portal pressure, stent patency rate during follow-up, and postoperative rebleeding rate, but these findings should be identified by further studies because of the small simple size. Because of these limitations, we call for future trials to include prospective, multicenter, randomized, blind design to verify our results.

\section{Conclusion}

In conclusion, our meta-analysis demonstrated that TIPS reduced the incidence of variceal rebleeding, and TIPS achieved ideal efficacy and safety in the treatment of PVCT, so PVCT should not be considered as an absolute contraindication of TIPS. What is more, although TIPS is still technically challenging, it remains an option for patients with PVCT.

\section{Acknowledgment}

The authors would like to express their deepest gratitude to K. Hatakeyama, a doctor from Niigata City, Japan, for his helpful suggestions and support in the revised manuscript.

\section{Statement of Ethics}

The article does not contain any studies with human participants or animals performed by any of the authors. Therefore, the paper is exempt from further Ethical Committee approval.

\section{Conflict of Interest Statement}

None of the authors have identified any conflicts of interest and financial disclosures.

\section{Funding Sources}

This research received no external funding.

\section{Author Contributions}

Conceptualization: Jingxin Yan and Lushun Zhang; validation: Yaxuan Wang, Meilin Zhu, Jingxin Yan, and Lushun Zhang; writing - original draft preparation: Jingxin Yan, Manjun Deng, Yaxuan Wang, Meilin Zhu, Ting Li; writing - review and editing: Haiyang Hu, Zhenwu Lei, Yaxuan Wang, Yingxing Guo, Lushun Zhang; software: Jingxin Yan and Manjun Deng.

\section{Data Availability Statement}

All data were derived from previously published studies, so the data availability statement is not required.

\section{References}

1 DeLeve LD, Valla DC, Garcia-Tsao G; American Association for the Study Liver Diseases. Vascular disorders of the liver. Hepatology. 2009;49(5):1729-64.

2 Kuy S, Dua A, Rieland J, Cronin DC 2nd. Cavernous transformation of the portal vein. ) Vasc Surg. 2016;63(2):529.

3 Condat B, Pessione F, Helene Denninger M, Hillaire S, Valla D. Recent portal or mesenteric venous thrombosis: increased recognition and frequent recanalization on anticoagulant therapy. Hepatology. 2000;32(3): 466-70.

4 Condat B, Pessione F, Hillaire S, Denninger M, Guillin M, Poliquin M, et al. Current outcome of portal vein thrombosis in adults: risk and benefit of anticoagulant therapy. Gastroenterology. 2001;120(2):490-7. 
5 Garcia-Pagan JC, Caca K, Bureau C, Laleman W, Appenrodt B, Luca A, et al. Early use of TIPS in patients with cirrhosis and variceal bleeding. N Engl J Med. 2010;362(25):2370-9.

6 Vizzutti F, Schepis F, Arena U, Laleman W, Appenrodt B, Luca A, et al. Transjugular intrahepatic portosystemic shunt (TIPS): current indications and strategies to improve the outcomes. Intern Emerg Med. 2020;15(1):37-48.

7 Cardenas A, Mendez-Bocanegra A. Report of the Baveno VI Consensus Workshop. Ann Hepatol. 2016;15(2):289-90.

8 Rodrigues SG, Sixt S, Abraldes JG, De Gottardi A, Klinger C, Bosch J, et al. Systematic review with meta-analysis: portal vein recanalisation and transjugular intrahepatic portosystemic shunt for portal vein thrombosis. Aliment Pharmacol Ther. 2019;49(1):20-30.

9 Tripathi D, Stanley AJ, Hayes PC, Travis S, Armstrong MJ, Tsochatzis EA, et al. Transjugular intrahepatic portosystemic stentshunt in the management of portal hypertension. Gut. 2020;69(7):1173-92.

10 Fanelli F. The evolution of transjugular intrahepatic portosystemic shunt: tips. ISRN Hepatol. 2014;2014:762096.

11 Moher D, Liberati A, Tetzlaff J, Altman DG; PRISMA Group. Preferred reporting items for systematic reviews and meta-analyses: the PRISMA statement. BMJ. 2009;339(7):b2535.

12 Stang A. Critical evaluation of the NewcastleOttawa scale for the assessment of the quality of nonrandomized studies in meta-analyses. Eur J Epidemiol. 2010;25(9):603-5.

13 Murad MH, Sultan S, Haffar S, Bazerbachi F. Methodological quality and synthesis of case series and case reports. BMJ Evid Based Med. 2018;23:60-3.

14 R Core Team. R: a language and environment for statistical computing. Vienna, Austria: R Foundation for Statistical Computing; 2017. Available from: http://www.R-project.org/ (accessed September 3, 2013).

15 Su YS, Yajima M. R2jags: using R to Run “JAGS". R package version 0.5-7. 2017. Available from: http://CRAN.R-project.org/ package $=$ R2jags. (accessed December 2, 2015).

16 Lambert PC, Sutton AJ, Burton PR, Abrams $\mathrm{KR}$, Jones $\mathrm{DR}$. How vague is vague? A simulation study of the impact of the use of vague prior distributions in MCMC using WinBUGS. Stat Med. 2005;24:2401-28.

17 Freeman MF, Tukey JW. Transformations related to the angular and the square root. Ann Math Statist. 1950;21(4):607-11.

18 Ferrari AJ, Somerville AJ, Baxter AJ, Norman $\mathrm{R}$, Patten SB, Vos T, et al. Global variation in the prevalence and incidence of major depressive disorder: a systematic review of the epidemiological literature. Psychol Med. 2013; 43:471-81.

19 Fanelli F, Angeloni S, Salvatori FM, Marzano C, Boatta E, Merli M, et al. Transjugular intrahepatic portosystemic shunt with expandedpolytetrafuoroethylene-covered stents in non-cirrhotic patients with portal cavernoma. Dig Liver Dis. 2011;43(1):78-84.
20 Guo FF, Wu ZY, Zhou PL, Han XW. Transjugular intrahepatic portosystemic shunt for the treatment cavernous transformation of the portal vein with vareceal bleeding. Zhonghua Yi Xue Za Zhi. 2020;100(5):387-90.

21 Li LN, Sun XY, Wang GC, Tian XG, Zhang MY, Jiang KT, et al. Transjugular intrahepatic portosystemic shunt prevents rebleeding in cirrhotic patients having cavernous transformation of the portal vein without improving their survival. J Dig Dis. 2019;20(2):89-96.

22 Luo X, Nie L, Zhou B, Yao D, Ma H, Jiang M, et al. Transjugular intrahepatic portosystemic shunt for the treatment of portal hypertension in noncirrhotic patients with portal cavernoma. Gastroenterol Res Pract. 2014;2014:659726.

23 Li MA, Luo JY, Zhang YY, Wu C, Qiang JS, Wang HF, et al. Percutanous transhepatic intrahepatic portosystemic shunt for chronic portal vein occlusion and cavernous transformation with symptomatic portal hypertension. Chin J Radiol. 2018;52(1):46-50.

24 Niu XK, Das SK, Wu HL, Chen Y. Computed tomography-based score model/nomogram for predicting technical and midterm outcomes in transjugular intrahepatic portosystemic shunt treatment for symptomatic portal cavernoma. World J Clin Cases. 2020;8(5): 887-99.

25 Qi X, Han G, Yin Z, He C, Wang J, Guo W, et al. Transjugular intrahepatic portosystemic shunt for portal cavernoma with symptomatic portal hypertension in non-cirrhotic patients. Dig Dis Sci. 2012;57(4):1072-82.

26 Senzolo M, Tibbals J, Cholongitas E, Triantos CK, Burroughs AK, Patch D. Transjugular intrahepatic portosystemic shunt for portal vein thrombosis with and without cavernous transformation. Aliment Pharmacol Ther. 2006;23(6):767-75.

27 Shen Q, Yang ZW, Jiang N, Huang N, Xie H. Transjugular intrahepatic portosystemic shunt for portal vein hypertension compicated from cavernous transformation of portal vein. Chin J Interv Imaging Ther. 2016; 13(11):658-11.

28 Jin SY, Wang GC, Li ZP, Huang GJ, Li C, Chen GH, et al. Transjugular intrahepatic portosystemic shunt for the prevention of gastroesophageal variceal rebleeding in patients with cavernous transformation of portal vein. Chin J Digest Med Imageol. 2021; 11(1):31-6.

29 de Franchis R; Baveno VI Faculty. Expanding consensus in portal hypertension: report of the Baveno VI Consensus Workshop stratifying risk and individualizing care for portal hypertension. J Hepatol. 2015;63(3): $743-52$.

30 Garcia-Tsao G, Abraldes JG, Berzigotti A, Bosch J. Portal hypertensive bleeding in cirrhosis: risk stratification, diagnosis, and management - 2016 practice guidance by the American Association for the study of liver diseases. Hepatology. 2017;65(1):310-35.
31 Boyer TD, Haskal ZJ; American Association for the Study of Liver Diseases. The role of transjugular intrahepatic portosystemic shunt in the management of portal hypertension. Hepatology. 2005;41(2):386-400.

32 Boyer TD, Haskal ZJ; American Association for the Study of Liver Diseases. The role of transjugular intrahepatic portosystemic shunt (TIPS) in the management of portal hypertension: update 2009. Hepatology. 2010; 51(1):306.

33 Kawamata H, Kumazaki T, Kanazawa H, Takahashi S, Tajima H, Hayashi H. Transjugular intrahepatic portosystemic shunt in a patient with cavernomatous portal vein occlusion. Cardiovasc Intervent Radiol. 2000; 23(2):145-9.

34 Thornburg B, Desai K, Hickey R, Hohlastos E, Kulik L, Ganger D, et al. Pretransplantation portal vein recanalization and transjugular intrahepatic portosystemic shunt creation for chronic portal vein thrombosis: final analysis of a 61-patient cohort. J Vasc Interv Radiol. 2017;28(12):1714-21.e2.

$35 \mathrm{Chu} H \mathrm{H}$, Kim HC, Jae HJ, Yi NJ, Lee KW, Suh KS, et al. Percutaneous transsplenic access to the portal vein for management of vascular complication in patients with chronic liver disease. Cardiovasc Intervent Radiol. 2012; 35(6):1388-95.

36 Qi X, Han G. Transjugular intrahepatic portosystemic shunt in the treatment of portal vein thrombosis: a critical review of literature. Hepatol Int. 2012;6(3):576-90.

37 Bilbao JI, Arias M, Herrero JI, Iglesias A, Martinez RF, Alejandre PL, et al. Percutaneous transhepatic treatment of a posttransplant portal vein thrombosis and a preexisting spontaneous splenorenal shunt. Cardiovasc Intervent Radiol. 1995;18(5):323-6.

38 Liang HL, Yang CF, Pan HB, Chen CK, Chang JM. Percutaneous transsplenic catheterization of the portal venous system. Acta Radiol. 1997;38(2):292-5.

39 Adani GL, Baccarani U, Risaliti A, Sponza M, Gasparini D, Bresadola F, et al. Percutaneous transhepatic portography for the treatment of early portal vein thrombosis after surgery. Cardiovasc Intervent Radiol. 2007;30(6): 1222-6.

40 Bertram H, Pfister ED, Becker T, Schoof S Transsplenic endovascular therapy of portal vein stenosis and subsequent complete portal vein thrombosis in a 2-year-old child. J Vasc Interv Radiol. 2010;21(11):1760-4.

41 Van Ha TG, Hodge J, Funaki B, Lorenz J, Rosenblum J, Straus C, et al. Transjugular intrahepatic portosystemic shunt placement in patients with cirrhosis and concomitant portal vein thrombosis. Cardiovasc Intervent $\mathrm{Ra}$ diol. 2006;29(5):785-90.

42 Luca A, Miraglia R, Caruso S, Milazzo M, Sapere C, Maruzzelli L, et al. Short- and longterm effects of the transjugular intrahepatic portosystemic shunt on portal vein thrombosis in patients with cirrhosis. Gut. 2011;60(6): 846-52. 
43 Luo X, Wang Z, Tsauo J, Zhou B, Zhang H, Li $\mathrm{X}$. Advanced cirrhosis combined with portal vein thrombosis: a randomized trial of TIPS versus endoscopic band ligation plus propranolol for the prevention of recurrent esophageal variceal bleeding. Radiology. 2015;276(1):286-93.

44 Wils A, van der Linden E, van Hoek B, Pattynama PM. Transjugular intrahepatic portosystemic shunt in patients with chronic portal vein occlusion and cavernous transformation J Clin Gastroenterol. 2009;43(10):982-4.

45 Han G, Qi X, He C, Yin Z, Wang J, Xia J, et al. Transjugular intrahepatic portosystemic shunt for portal vein thrombosis with symptomatic portal hypertension in liver cirrhosis. J Hepatol. 2011;54(1):78-88.

46 Qi X, Han G, He C, Yin Z, Zhang H, Wang J, et al. Transjugular intrahepatic portosystemic shunt may be superior to conservative therapy for variceal rebleeding in cirrhotic patients with non-tumoral portal vein thrombosis: a hypothesis. Med Sci Monit. 2012;18(8): HY37-41.

47 Senzolo M, Sartori TM, Rossetto V, Burra P, Cillo U, Boccagni P, et al. Prospective evaluation of anticoagulation and transjugular intrahepatic portosystemic shunt for the management of portal vein thrombosis in cirrhosis. Liver Int. 2012;32(6):919-27.
48 Werner KT, Sando S, Carey EJ, Vargas HE, Byrne TJ, Douglas DD, et al. Portal vein thrombosis in patients with end stage liver disease awaiting liver transplantation: outcome of anticoagulation. Dig Dis Sci. 2013; 58(6):1776-80.

49 Wang Z, Jiang MS, Zhang HL, Wen NN, Luo $\mathrm{XF}, \mathrm{Li} \mathrm{X}$, et al. Is post-TIPS anticoagulation therapy necessary in patients with cirrhosis and portal vein thrombosis? A randomized controlled trial. Radiology. 2016;279(3):943-51.

50 Angeloni S, Merli M, Salvatori FM, De Santis A, Fanelli F, Pepino D, et al. Polytetrafluoroethylene-covered stent grafts for TIPS procedure: 1-year patency and clinical results. Am J Gastroenterol. 2004;99(2):280-5.

51 Rossi P, Salvatori FM, Fanelli F, Bezzi M, Rossi M, Marcelli G, et al. Polytetrafluoroethylene-covered nitinol stent-graft for transjugular intrahepatic portosystemic shunt creation: 3-year experience. Radiology. 2004;231(3): 820-30.

52 Bureau C, Garcia Pagan JC, Layrargues GP, Metivier S, Bellot P, Perreault P, et al. Patency of stents covered with polytetrafluoroethylene in patients treated by transjugular intrahepatic portosystemic shunts: long-term results of a randomized multicentre study. Liver Int. 2007;27(6):742-7.
53 Riggio O, Angeloni S, Salvatori FM, De Santis A, Cerini F, Farcomeni A, et al. Incidence, natural history, and risk factors of hepatic encephalopathy after transjugular intrahepatic portosystemic shunt with polytetrafluoroethylene-covered stent grafts. Am J Gastroenterol. 2008; 103(11):2738-46.

54 Francoz C, Belghiti J, Vilgrain V, Sommacale $\mathrm{D}$, Paradis V, Condat B, et al. Splanchnic vein thrombosis in candidates for liver transplanta- tion: usefulness of screening and anticoagulation. Gut. 2005;54:691-7.

55 Ma J, Yan Z, Luo J, Liu Q, Wang J, Qiu S. Rational classification of portal vein thrombosis and its clinical significance. PLoS One. 2014; 9:e112501.

56 Shen Y, Ma W, Hang Y, Liu LL, Jiang W, Wu $\mathrm{SD}$. Clinical application of liver stiffness measurement in patients with cavernous transformation of portal vein. Exp Ther Med. 2021; 21(5):442

57 Erman A, Sathya S, Nam A, Bielecki JM, Feld JJ, Thein HH, et al. Estimating chronic hepatitis $\mathrm{C}$ prognosis using transient elastography-based liver stiffness: a systematic review and meta-analysis. J Viral Hepat. 2018;25(5): 502-13.

58 Agbim U, Asrani SK. Non-invasive assessment of liver fibrosis and prognosis: an update on serum and elastography markers. Expert Rev Gastroenterol Hepatol. 2019;13(4): $361-74$ 